\title{
Ising model in small-world networks
}

\author{
Carlos P. Herrero \\ Instituto de Ciencia de Materiales, Consejo Superior de Investigaciones Científicas (C.S.I.C.), Campus de Cantoblanco, \\ 28049 Madrid, Spain \\ (October 27, 2018)
}

\begin{abstract}
The Ising model in small-world networks generated from two- and three-dimensional regular lattices has been studied. Monte Carlo simulations were carried out to characterize the ferromagnetic transition appearing in these systems. In the thermodynamic limit, the phase transition has a mean-field character for any finite value of the rewiring probability $p$, which measures the disorder strength of a given network. For small values of $p$, both the transition temperature and critical energy change with $p$ as a power law. In the limit $p \rightarrow 0$, the heat capacity at the transition temperature diverges logarithmically in two-dimensional (2D) networks and as a power law in 3D.
\end{abstract}

\section{INTRODUCTION}

Complex networks describe many systems in nature and society, and have been modeled traditionally by random graphs [1]. In the last years, new models of complex networks have been introduced, motivated by empirical data in different fields [2. In particular, smallworld networks have been studied, as they are suitable to describe properties of physical systems with underlying networks ranging from regular lattices to random graphs, by changing a single parameter [3]. Watts and Strogatz [4i proposed a model for this kind of networks, which is based on a locally highly connected regular lattice, in which a fraction $p$ of the links between nearest-neighbor sites are randomly replaced by new random links, thus creating long-range "shortcuts". The networks so generated are suitable to study different kinds of physical systems, such as neural networks [5] and man-made communication and transportation systems [4, 6 , 8].

These small-world networks interpolate between the two limiting cases of regular lattices $(p=0)$ and random graphs $(p=1)$. In the small-world regime, a local neighborhood is preserved (as for regular lattices), and at the same time some global properties of random graphs are maintained. The small-world effect is usually measured by the scaling behavior of the characteristic path length $\ell$, defined as the average of the shortest distance between two sites. For a random network one has a logarithmic increase of $\ell$ with the network size $N$ (i.e., the number of sites), while for a $d$-dimensional regular lattice one expects an algebraic increase: $\ell \sim N^{1 / d}$. In contrast, for a small-world network, $\ell$ follows the scaling law 9 11]

$$
\ell(N, p) \sim\left(N^{*}\right)^{1 / d} F\left(N / N^{*}\right),
$$

where the scaling function $F(u)$ has the limits $F(u) \sim$ $u^{1 / d}$ for $u \ll 1$ and $F(u) \sim \ln u$ for $u \gg 1 ; N^{*} \sim p^{-1}$ is a crossover size that separates the large- and small-world regimes [9,12,13]. This indicates that the small-world behavior appears for any finite value of $p(0<p<1)$ as soon as the network is large enough, and in particular the global characteristics of the network are changed dramatically in the presence of only a small fraction of shortcuts.

The importance of this shorter global length scale has been studied for several statistical physical problems on small-world networks. Among these problems, one finds in the literature the signal propagation speed [4], the spread of infections 114,15], as well as site and bond percolation 15 17]. The localization-delocalization transition of electron states has been also studied on quantum small-world networks 18$]$.

Up to now, most of the published work on small worlds has concentrated on networks obtained from onedimensional lattices. Barrat and Weight [10] and Gitterman 19] have studied the crossover from 1D to meanfield behavior for the ferromagnetic Ising model, which presents a phase transition of mean-field type for any value of the rewiring probability $p>0$, provided that the system size is large enough. Close to $p=0$ the transition temperature $T_{c}$ goes to zero as $1 /|\log p|$. A meanfield-type behavior has been also found for the XY model in small-world networks generated from one-dimensional chains [20].

More recently, Svenson and Johnston [21] have studied the damage spreading for Ising models on small-world networks obtained by rewiring two-dimensional (2D) and three-dimensional (3D) regular lattices. They found that these networks are more suitable than regular lattices to study social systems with the Ising model.

Here we investigate the ferromagnetic transition for the Ising model in small-world networks generated by rewiring $2 \mathrm{D}$ and $3 \mathrm{D}$ lattices. Contrary to the networks generated from 1D lattices, now one has phase transitions at finite temperatures $T_{c}>0$ for $p=0$. This means that one expects a change from an Ising-type transition at $p=0$ to a mean-field-type one in the small-world regime. We employ Monte Carlo (MC) simulations to obtain av- 


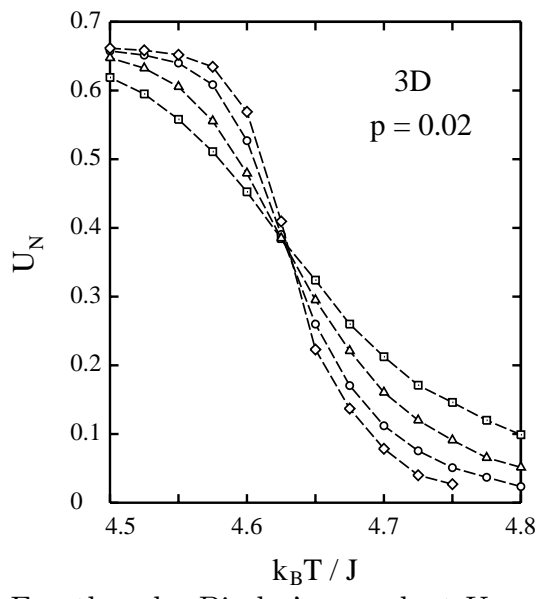

FIG. 1. Fourth-order Binder's cumulant $U_{N}$ as a function of temperature for small-world networks generated from 3D cubic lattices with rewiring probability $p=0.02$. Symbols represent different system sizes $N=L^{3}$ : squares, $L=15$; triangles, $L=20$; circles, $L=25$; diamonds, $L=30$.

erage magnitudes for finite-size systems. The resulting quantities are extrapolated to the thermodynamic (infinite size) limit, where the small-world behavior is expected to dominate the thermodynamic properties for any finite value of the rewiring probability $p>0$.

In Sec. II we describe the computational method. In Sec. III we present results of the MC simulations along with a discussion. The paper closes with some concluding remarks in Sec. IV.

\section{COMPUTATIONAL METHOD}

We consider the Hamiltonian:

$$
H=-\sum_{i<j} J_{i j} S_{i} S_{j},
$$

where $S_{i}= \pm 1(i=1, \ldots, N)$, and the coupling matrix $J_{i j}$ is given by

$$
J_{i j} \equiv \begin{cases}J(>0), & \text { if } i \text { and } j \text { are connected, } \\ 0, & \text { otherwise. }\end{cases}
$$

Monte Carlo simulations have been carried out on networks of different sizes, generated from the 2D square and 3D cubic lattices. Small-world networks were generated by randomly replacing a fraction $p$ of the links of the regular lattices with new random connections. This procedure keeps constant the total number of links in the rewired networks. Thus, the average coordination number $z$ in the 2D and 3D cases amounts to 4 and 6 , respectively. In the rewiring process we avoided isolated sites (with zero links). With this procedure we obtained networks in which more than $99.9 \%$ of the sites were connected in a single component (Note that a random graph has usually many components of various sizes). The remaining sites (when they appeared) were excluded from

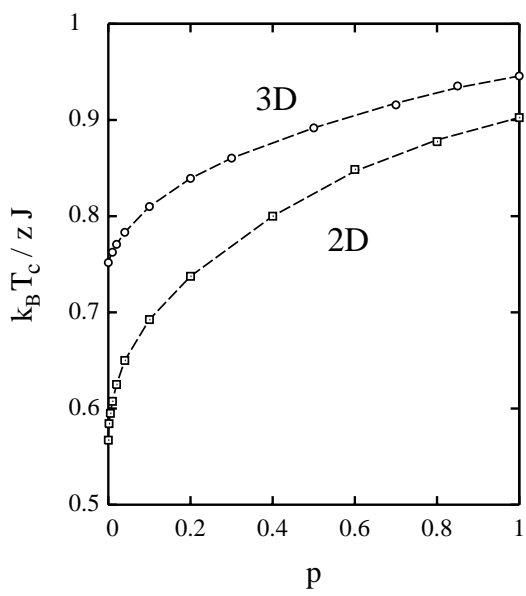

FIG. 2. Transition temperature $T_{c}$, normalized by the average coordination number $z$, as a function of the rewiring probability $p$ for small-world networks generated from 2D and 3D lattices. Dashed lines are guides to the eye.

the final networks employed for the MC simulations. The size of the networks used in our calculations was larger than the crossover size $\left.N^{*} 13,16\right]$, so that we were in the small-world regime.

The largest networks employed here included $200 \times$ 200 sites for the 2 D system and $40 \times 40 \times 40$ sites for the $3 \mathrm{D}$ network. Periodic boundary conditions were assumed. Sampling of the configuration space has been carried out by the Metropolis local update algorithm 22. Several thermodynamic quantities and moments of the order parameter have been calculated for different simulation-cell sizes. Finite-size scaling was then employed to obtain the magnitudes corresponding to the thermodynamic limit (extrapolation to infinite size). In the remainder of the paper, the presented values for the different quantities will correspond to the extrapolated ones, unless explicit mention is made indicating a particular network size.

The ferromagnetic transition temperature has been determined by using Binder's fourth-order cumulant [22]

$$
U_{N}(T) \equiv 1-\frac{\left\langle M^{4}\right\rangle_{N}}{3\left\langle M^{2}\right\rangle_{N}^{2}}
$$

where the magnetization $M$ of a given spin configuration is given by $M=\sum_{i=1}^{N} S_{i} / N$. As an example, we present in Fig. 1 the cumulant $U_{N}$ as a function of temperature for different system sizes in the 3D case for $p=0.02$. The transition temperature is obtained from the unique crossing point for the different sizes $N$.

The heat capacity per site, $c_{v}$, was obtained from the energy fluctuations at a given temperature, by using the expression

$$
c_{v}=\frac{(\Delta E)^{2}}{N k_{B} T^{2}},
$$

where $(\Delta E)^{2}=\left\langle E^{2}\right\rangle-\langle E\rangle^{2}$. 


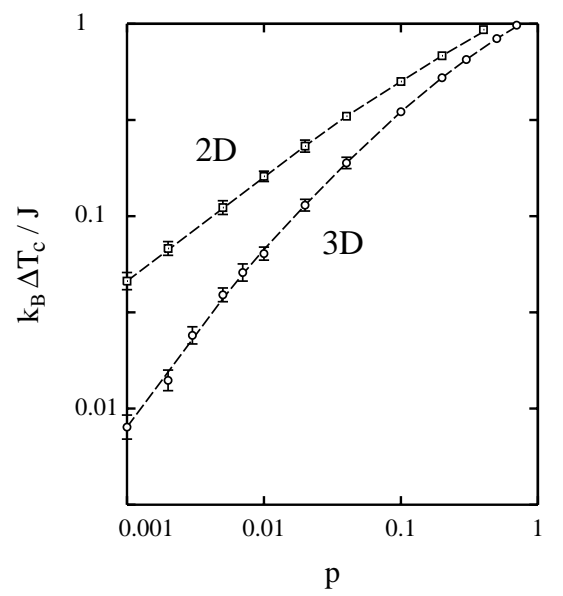

FIG. 3. Dependence upon the rewiring probability $p$ of the shift in transition temperature $\Delta T_{c}$ with respect to the regular lattices, for $2 \mathrm{D}$ and $3 \mathrm{D}$ networks. Lines are guides to the eye.

\section{RESULTS AND DISCUSSION}

In Fig. 2, we present the transition temperature $T_{c}$ as a function of the rewiring probability $p$ for $2 \mathrm{D}$ and $3 \mathrm{D}$ networks. Similar to the $1 \mathrm{D}$ case 10 , $T_{c}$ changes fast close to $p=0$, and the derivative $d T_{c} / d p$ becomes smaller as $p$ increases. However, the sharp change of $T_{c}$ for small $p$ shows a behavior different than that found in the $1 \mathrm{D}$ case, where $T_{c} \sim|\log p|^{-1}$. In the limit $p=1$ one finds an increase in $T_{c}$ as $z$ rises from 4 (in 2D) to 6 (in $3 \mathrm{D}$ ), as expected for random lattices, for which one has $k_{B} T_{c} / z J \rightarrow 1$ as $z \rightarrow \infty$.

To analyze the change in critical temperature with $p$, we call $\Delta T_{c}=T_{c}-T_{c}^{0}$, being $T_{c}^{0}$ the transition temperature for the corresponding $2 \mathrm{D}$ or $3 \mathrm{D}$ regular lattice. In Fig. 3 we show the dependence of $\Delta T_{c}$ upon $p$ for the $2 \mathrm{D}$ and $3 \mathrm{D}$ cases in a log-log plot. In both cases we find that $\Delta T_{c}$ can be fitted by a power law $\Delta T_{c} \sim p^{s}$ for $p \lesssim 0.01$. The exponent $s$ is $0.52 \pm 0.03$ for $2 \mathrm{D}$ and $0.96 \pm 0.04$ for $3 \mathrm{D}$. Our result for $2 \mathrm{D}$ networks is compatible with a $\sqrt{p}$ dependence for $\Delta T_{c}$ near $p=0$, which means that the derivative $d T_{c} / d p$ diverges as $\sim 1 / \sqrt{p}$ for $p \rightarrow 0$. In the $3 \mathrm{D}$ case, our results seem to indicate a dependence $\Delta T_{c} \sim p$ for small $p$. However, in this case the lowest $p$ values studied here may still be too high to attain the small- $p$ regime (see below).

Associated with the increase in $T_{c}$ as the rewiring probability $p$ rises, one expects an increase in the critical energy $E\left(T_{c}\right)$. We call $e_{c}=E\left(T_{c}\right) / N$ the critical energy per site, and $\Delta e_{c}=e_{c}-e_{c}^{0}$ its change with respect to the regular lattice $(p=0)$. This difference $\Delta e_{c}$ is shown in Fig. 4 as a function of $p$ for 2D (squares) and 3D (circles) networks, in a log-log plot. For $p \lesssim 0.01, \Delta e_{c}$ can be well fitted in both cases by a power law of the form $\Delta e_{c} \sim p^{u}$. For the exponent $u$, we find $u=0.43 \pm 0.03$ and $0.56 \pm 0.04$ in $2 \mathrm{D}$ and $3 \mathrm{D}$, respectively.

A characterization of the ferromagnetic phase transi-

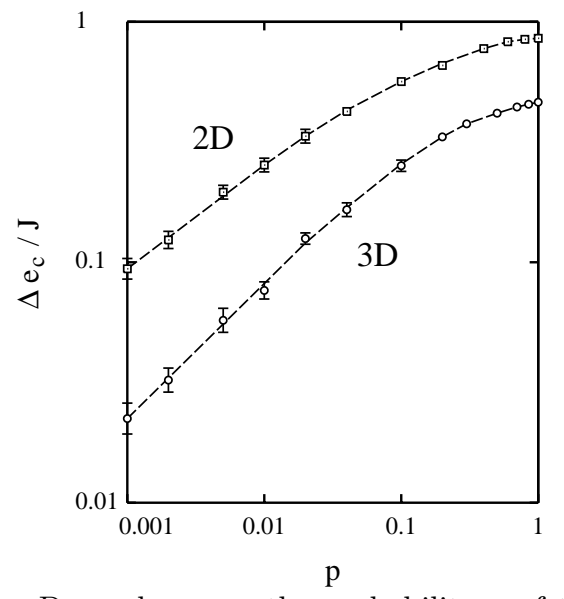

FIG. 4. Dependence on the probability $p$ of the shift in critical energy per site $\Delta e_{c}$ with respect to the regular lattices, for $2 \mathrm{D}$ and $3 \mathrm{D}$ networks. Lines are guides to the eye.

tion in these networks requires the determination of the universality class to which it corresponds. In the limit $p=0$ (regular lattices), one has transitions of the $2 \mathrm{D}$ and 3D Ising type. In order to determine the type of the phase transition at $p>0$, we have studied the critical exponent $\beta$, which gives the temperature dependence of the order parameter close to the transition temperature: $\langle M\rangle \sim\left(T_{c}-T\right)^{\beta}$ for $T<T_{c}$. For the different values of the rewiring probability $p$ studied here, we have calculated numerically the logarithmic derivative

$$
\mu(t)=\frac{d \log \langle M\rangle}{d \log t}
$$

for $t=T_{c}-T>0$, which is related to the exponent $\beta$ through the limit $\beta=\lim _{t \rightarrow 0} \mu(t)$.

In Fig. 5 we present results for the derivative $\mu$ as a function of temperature for several values of $p$ and for a $2 \mathrm{D}$ network of size $200 \times 200$. For reference, we also present results of MC simulations for $p=0$ (Ising model on a regular $2 \mathrm{D}$ lattice) for the same system size, which converge to $\beta=0.125$, the critical exponent for the $2 \mathrm{D}$ Ising model. In all cases $p>0$, the extrapolation $T \rightarrow T_{c}$ gives an exponent $\beta$ close to 0.5 , the value corresponding to a mean-field-type transition. However, for decreasing $p$, the observation of the mean-field character of the transition requires to go to temperatures closer to $T_{c}$ (or equivalently to larger system sizes; see 22]). Thus, for $p=0.001$ we are still far from the value 0.5 at the temperatures at which the employed system size allows us to give a precise value for the order parameter $\langle M\rangle$. But even in this case, the departure from the value expected for a $2 \mathrm{D}$ Ising-type transition is clear close to $T_{c}$.

A complementary way to confirm the mean-field character of the phase transition consists in determining the exponent $\nu$, which controls the behavior of the correlation length near the critical temperature:

$$
\xi \sim\left|T-T_{c}\right|^{-\nu}
$$




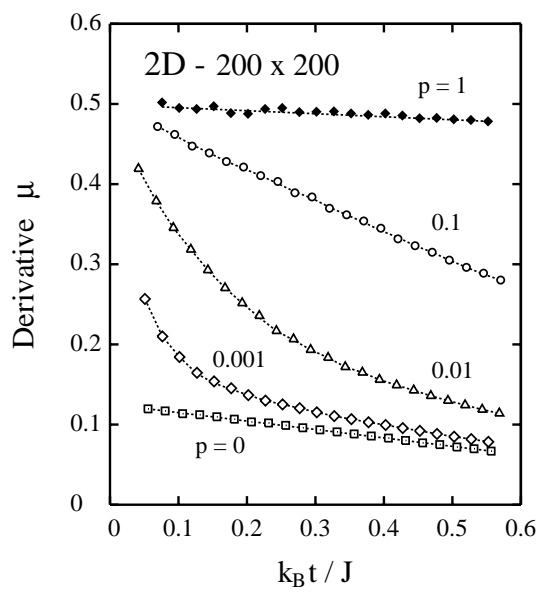

FIG. 5. Logarithmic derivative $\mu$ versus the temperature difference $t=T_{c}-T$ for small-world networks generated by rewiring a $2 \mathrm{D}$ lattice of size $200 \times 200$. Different symbols represent results obtained for several values of the rewiring probability $p$. From top to bottom: $p=1,0.1,0.01,0.001$, and 0 .

Close to $T_{c}$, this critical exponent is related to the temperature dependence of the fourth-order cumulant defined in Eq. (4) as 20,22]:

$$
U_{N}(T) \approx U^{*}+U_{1}\left(1-\frac{T}{T_{c}}\right) N^{1 / \nu}
$$

with $U^{*}$ and $U_{1}$ independent of $T$ and the system size $N$. From this expression we have:

$$
\frac{\Delta U_{N}}{\Delta T} \propto-N^{1 / \nu}
$$

which allows us to calculate the exponent $\nu$ from the cumulant $U_{N}$ derived from the MC simulations. The values of $\nu$ obtained by this method agreed in all cases (within error bars) with the critical exponent corresponding to mean-field transitions: $\nu=0.5$.

We conclude that the mean-field character of the phase transition (which is the one found in random networks) should appear in the thermodynamic limit for any $p>$ 0 . This is in line with the observation mentioned above that the characteristics of the random graphs (e.g., mean distance between sites $l \sim \log N$ ) show up in small-world networks as soon as their size $N$ is large enough.

To understand the dependence on $p$ of the transition temperature $T_{c}$ for small $p$, we will consider the two length scales present in this problem. On one side we have the correlation length $\xi$, which near $T_{c}$ follows the temperature dependence given in Eq. (7). On the other side, we have a length scale characteristic of the smallworld network, given by the typical distance between ends of shortcuts: $\zeta=(p z)^{-1 / d}$ [16]. When the correlation length is smaller than $\zeta$, the system behaves basically as a regular lattice. When $\xi$ grows beyond $\zeta$ (as happens when we approach the transition temperature

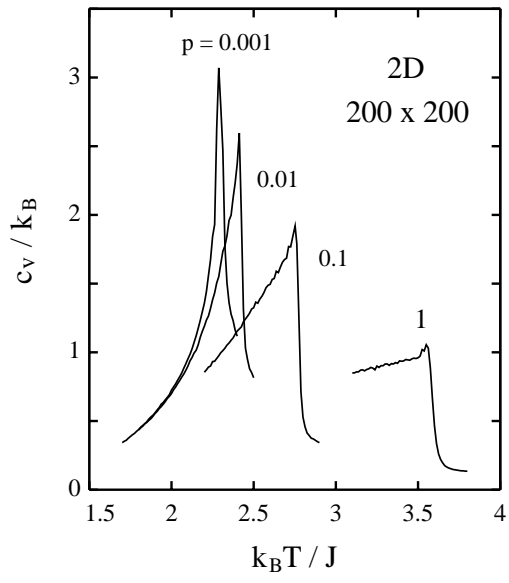

FIG. 6. Heat capacity per site $c_{v}$ versus temperature for small-world networks generated from a $2 \mathrm{D}$ lattice of size $200 \times 200$. The plotted curves correspond to different values of the rewiring probability $p$, as indicated by the labels.

from above, $T \rightarrow T_{c}^{+}$), the "long-range" interactions introduced by the shortcuts come into play and give rise to the mean-field behavior. Thus, the transition between the regular-lattice behavior and the mean-field one occurs for $\xi \approx \zeta$. For the 1D Ising model, the correlation length at low temperatures $\left(k_{B} T \ll J\right)$ is given by $\xi \sim \exp \left(2 J / k_{B} T\right)$ 24. Taking into account that in this case $\zeta \sim 1 / p$, the condition $\xi \approx \zeta$ suggests a critical temperature for the $1 \mathrm{D}$ small-world $T_{c} \sim|\log p|^{-1}$, in agreement with more detailed calculations for this system [10]. For the 2D Ising model the correlation length near $T_{c}$ scales as $\xi \sim\left|T-T_{c}^{0}\right|^{-1}$ [23,24], whereas now $\zeta \sim 1 / p^{1 / 2}$. Then, using the same argument, one expects for small-world networks generated from $2 \mathrm{D}$ lattices: $T_{c}-T_{c}^{0} \sim p^{1 / 2}$, in good agreement with the results of our Monte Carlo simulations (see Fig. 3). Thus, it seems that in general, for systems with a correlation length diverging as $\left|T-T_{c}^{0}\right|^{-\nu}$, the order-disorder transition temperature for small $p$ depends on the rewiring probability as

$$
T_{c}-T_{c}^{0} \sim p^{1 / \nu d} .
$$

Note that here $\nu$ is the critical exponent of the considered model in the regular lattice, not the one corresponding to the phase transition in the small-world network (which is the mean-field one). Hence, we see that the condition $\xi \approx$ $\zeta$ describes the transition between the large-world regime (which corresponds to regular lattices) and the smallworld one 13], as well as the ferromagnetic transition, indicating that the small-world transition and the orderdisorder one are intimately related.

The above argument, however, seems to fail for smallworld networks built up from 3D lattices. For the 3D Ising model one has a critical exponent $\nu \approx 0.63$ [24], which should give for small $p$ a dependence: $\Delta T_{c} \sim p^{5.53}$, with an exponent clearly smaller than that derived from our MC simulations, where we found for $p \lesssim 0.01$ : 
$\Delta T_{c} \sim p^{0.96}$. It seems that this discrepancy appears because the smallest $p$ value employed in our simulations $\left(p=10^{-3}\right)$ is still too large to observe the small- $p$ behavior. Although for such values of $p$ we are clearly in the small-world regime, the corresponding value of $\zeta$ in $3 \mathrm{D}(\zeta \sim 5)$, is too small to allow the correlation length to reach the critical dependence of the Ising model in the regular lattice: $\xi \sim\left|T-T_{c}^{0}\right|^{-\nu}$. In other words, to observe the $p$-dependence of $T_{c}$ given in Eq. (10), one needs $\zeta$ values larger (i.e., smaller $p$ values or larger networks) than those employed here. A similar conclusion was proposed for the XY model on 1D small-world networks [20], for which MC simulations gave a dependence $T_{c} \approx a \log p+b$ (with $a$ and $b$ numerical constants) instead of $T_{c} \sim p$, expected from the above argument.

For mean-field-type transitions, the heat capacity per site $c_{v}$ shows a finite jump (not a divergence) at $T_{c}$. Given that at $p=0$ one has Ising-type phase transitions with divergent heat capacity at $T_{c}$, such a jump in $c_{v}$ will diverge in the limit $p \rightarrow 0$. The temperature dependence of $c_{v}$ is displayed in Fig. 6 for several values of $p$ and for networks built up from a $200 \times 2002$ D lattice. As expected, one observes an increase in the maximum value of $c_{v}$ as $p$ is reduced.

The maximum value of $c_{v}$ for each $p$, extrapolated to the thermodynamic limit, is shown in Fig. 7 for $2 \mathrm{D}$ and 3D small-world networks. In Fig. 7(a) we present our results for $2 \mathrm{D}$, which for small $p$ display a logarithmic dependence $c_{v}^{m}=a-b \ln p$, with the numerical constants $a=1.74 \pm 0.06$ and $b=0.20 \pm 0.01$. This logarithmic dependence of the heat capacity can be explained by arguments similar to those employed above to analyze the behavior of the transition temperature as $p \rightarrow 0$. For the Ising model in $2 \mathrm{D}$ regular lattices, the heat capacity near $T_{c}^{0}$ diverges as: $c_{v}(T) \sim-\log \left|T-T_{c}^{0}\right|[23]$. Taking into account the relation between $T_{c}$ and $p$ given above for small $p$, one finds for the $2 \mathrm{D}$ small-world networks $c_{v}^{m} \sim-\log p$, in agreement with the results of our Monte Carlo simulations.

In the $3 \mathrm{D}$ Ising model, $c_{v}$ has at $T_{c}^{0}$ a singularity of the form $\left|T-T_{c}^{0}\right|^{-\alpha}$, with $\alpha \approx 0.12$ 24]. Assuming that $T_{c}-T_{c}^{0} \sim p^{1 / \nu d}$, as in Eq. (10), one expects for $c_{v}^{m}$ close to $p=0$ a power-law of the form: $c_{v}^{m} \sim p^{w}$, with an exponent $w=-\alpha / \nu d=-0.063$. In Fig. $7(\mathrm{~b})$ we present the values of $c_{v}^{m}$ derived from our MC simulations as a function of $p$ in a log-log plot. These results are consistent with a power-law dependence $c_{v}^{m} \sim p^{w}$ at small $p$. In fact, for $p \lesssim 0.01$ our results can be fitted with an exponent $w=-0.057 \pm 0.005$. However, as in the case of the transition temperature discussed above, smaller values of $p$ are necessary to determine unambigously this dependence from numerical simulations, and in particular to find the exponent $w$.
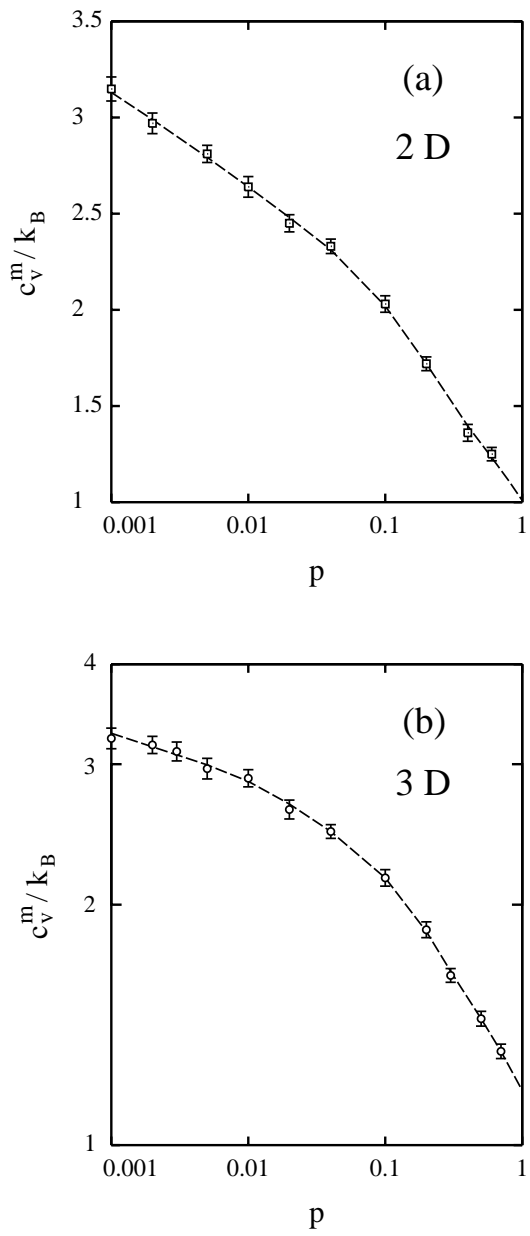

FIG. 7. Maximum value of the heat capacity per site $c_{v}^{m}$ as a function of the rewiring probability $p$. Symbols correspond to the extrapolation of finite-size results to $N \rightarrow \infty$. (a) $2 \mathrm{D}$ networks, in a semi-logarithmic plot; (b) 3D networks, in a log-log plot. Dashed lines are guides to the eye. 


\section{CONCLUDING REMARKS}

We have studied the ferromagnetic transition that appears for the Ising model in small-world networks generated from $2 \mathrm{D}$ and $3 \mathrm{D}$ regular lattices. In these networks, the presence of a small disorder $(p>0)$ causes a change in the universality class of the order-disorder transition, from Ising for $p=0$ to mean-field type for $p>0$.

Our results indicate that the order-disorder transition occurs at a temperature $T_{c}$ where the spin correlation length $\xi$ is on the order of the length $\zeta$ (typical distance between ends of shortcuts), characteristic of these networks. In particular, close to $p=0$ this gives a dependence $T_{c} \sim|\log p|^{-1}$ in $1 \mathrm{D}$ networks, and $T_{c}-T_{c}^{0} \sim p^{1 / \nu d}$ for networks generated from regular lattices of higher dimensions. This is the dependence found from our Monte Carlo simulations for $2 \mathrm{D}$ networks. In the $3 \mathrm{D}$ case we find a power law for $T_{c}-T_{c}^{0}$, but the determination of the actual exponent from MC simulations for $p \rightarrow 0$ requires $p$ values smaller (i.e., networks larger) than those employed here.

From the results presented in this paper, it is clear that there is a close relation between the small-world transition and the order-disorder transition in this kind of networks.

The author benefited from useful discussions with M. A. R. de Cara and M. Saboya. Thanks are due to E. Chacón for critically reading the manuscript. This work was supported by CICYT (Spain) under Contract No. PB96-0874, and by DGESIC through Project No. 1FD97-1358.

[1] B. Bollobás, Random Graphs (Academic Press, New York, 1985).

[2] S. H. Strogatz, Nature 410, 268 (2001); R. Albert and A. L. Barabási, preprint cond-mat/0106096; S. N. Dorogovtsev and J. F. F. Mendes, preprint cond-mat/0106144

[3] D. J. Watts, Small Worlds (Princeton University Press, Princeton, 1999).

[4] D. J. Watts and S. H. Strogatz, Nature 393, 440 (1998).

[5] L. F. Lago-Fernández, R. Huerta, F. Corbacho, and J. A. Sigüenza, Phys. Rev. Lett. 84, 2758 (2000).

[6] V. Latora and M. Marchiori, Phys. Rev. Lett. 87, 198701 (2001).

[7] M. E. J. Newman, J. Stat. Phys. 101, 819 (2000).

[8] R. Albert, H. Jeong, and A. L. Barabási, Nature 401, 130 (1999); A. L. Barabási and R. Albert, Science 286, 509 (1999).

[9] M. Barthélémy and L. A. N. Amaral, Phys. Rev. Lett. 82, 3180 (1999); ibid, 5180 (1999).

[10] A. Barrat and M. Weigt, Eur. Phys. J. B 13, 547 (2000).

[11] A. Scala, L. A. N. Amaral, and M. Barthélémy, Europhys. Lett. 55, 594 (2001).
[12] A. Barrat, preprint cond-mat/9903323.

[13] M. A. de Menezes, C. F. Moukarzel, and T. J. P. Penna, Europhys. Lett. 50, 574 (2000).

[14] M. Kuperman and G. Abramson, Phys. Rev. Lett. 86, 2909 (2001).

[15] C. Moore and M. E. J. Newman, Phys. Rev. E 61, 5678 (2000).

[16] M. E. J. Newman and D. J. Watts, Phys. Rev. E 60, 7332 (1999).

[17] C. Moore and M. E. J. Newman, Phys. Rev. E 62, 7059 (2000).

[18] C. P. Zhu and S. J. Xiong, Phys. Rev. B 62, 14780 (2000); 63, 193405 (2001).

[19] M. Gitterman, J. Phys. A: Math. Gen. 33, 8373 (2000).

[20] B. J. Kim, H. Hong, P. Holme, G. S. Jeon, P. Minnhagen, and M.Y. Choi, Phys. Rev. E 64, 056135 (2001).

[21] P. Svenson and D. A. Johnston, Phys. Rev. E. 65, 036105 (2002).

[22] K. Binder and D.W. Heermann, Monte Carlo Simulation in Statistical Physics, 3rd ed. (Springer-Verlag, Berlin, 1997).

[23] B. M. McCoy and T. T. Wu, The Two-Dimensional Ising Model (Harvard University, Cambridge, Massachusetts, 1973).

[24] J. J. Binney, N. J. Dowrick, A. J. Fisher, and M. E. J. Newman, The Theory of Critical Phenomena (Oxford University, Oxford, 1992). 